\title{
NUTRIÇÃO MINERAL DAS PLANTAS ORNAMENTAIS III. ABSORÇÃO DE NUTRIENTES PELA RAINHA MARGARIDA (Callestephus chinensis) *
}

\author{
H. P. HAAG \\ G. D. DE Oliveira \\ S. Watanabe \\ P. D. Fernandes
}

\section{RESUMO}

\begin{abstract}
No sentido de aquilatar a extração dos macro e micronutrientes, com exceção do $\mathrm{C} 1$ e Mo, aliada ao crescimento da planta, amostragens de rainha margarida (Callestephus chinensis) foram executadas aos $0,18,34,46,59$ e 77 dias após o transplante. As plantas foram divididas em raiz, caule, folhas, botões florais, flores analisadas para $\mathrm{N}, \mathrm{P}, \mathrm{K}, \mathrm{Ca}, \mathrm{Mg}, \mathrm{S}, \mathrm{B}, \mathrm{Cu}, \mathrm{Fe}, \mathrm{Mn}$ e $\mathrm{Zn}$. Observou-se que o crescimento da rainha margarida é contínuo, acentuando-se após os 59 dias de transplante. O teor porcentual dos nutrientes aos 34-46 dias, na matéria seca, oscilou em torno de $4,09 \%-4,40 \%$ para $N ; 0,44 \%$ $-0,46 \%$ para $\mathrm{P} ; 1,65 \%$ - 3,19\% para $\mathrm{K} ; 1,01 \%-1,10 \%$ para $\mathrm{Ca}$; $0,34 \%-0,45 \%$ para $\mathrm{Mg} ; 0,42 \%$ - $0,43 \%$ para $\mathrm{S}$. Para os micronutrientes os valores encontrados, na mesma época, foram em ppm: $\mathrm{B}-23-36 ; \mathrm{Cu}-18-20 ; \mathrm{Fe}-105-150 ; \mathrm{Mn}-115-135 ; \mathrm{Zn}-64-111$. Uma planta de rainha-margarida aos 77 dias contem: $2.049,9 \mathrm{mg}$ de $\mathrm{N} ; 212,5 \mathrm{mg}$ de $\mathrm{P} ; 2.496,6 \mathrm{mg}$ de $\mathrm{K} ; 915,7 \mathrm{mg}$ de $\mathrm{Ca} ; 356,6 \mathrm{mg}$ de $\mathrm{Mg} ; 159,1 \mathrm{mg}$ de $\mathrm{S} ; 2.140 \mathrm{ug}$ de $\mathrm{B} ; 3.070$ ug de $\mathrm{Cu} ; 17.142$ ug de Fe; $6.946 \mathrm{ug}$ de $\mathrm{Mn} ; 3.931 \mathrm{ug}$ de $\mathrm{Zn}$.
\end{abstract}

\section{INTRODUÇAO}

A exploração de cultura de flores é intensamente desenvolvida e explorada em vários países como Holanda, França, Alemanha, Japão e Estados Unidos.

\footnotetext{
- Entregue para publicação em 28/11/1974.

* Dep. de Química, E. S. A. «Luiz de Queiroz», USP. Piracícaba.

*** Colégio Agrícola de São Manuel, S. P.

**** Dep. de Fitotecnia, Fac. Med. Vet. e Agr. de Jaboticabal, SP.
} 
No Brasil essa exploração vem tomando grande impulso nestes últimos anos, apesar da ausência quase total de pesquisa.

0 comércio interno baseia-se principalmente em flores de corte, tendo lugar de destaque a rosa, o cravo e o gladíolo (MIRANDA, s/ data). São deveras limitados os trabalhos que tratam da nutrição mineral destas espécies, podendo-se citar HAAG et al (1970), FERNANDES et al. (1971), FERNANDES (1974) entre outros.

A rainha margarida se caracteriza pela facilidade de cultivo e boa aceitação pelo público. O consumo desta flor, oscila em torno de 83.000 maços por ano no CEAGESP, situado na capital do Estado de São Paulo, ocupando o $6 .^{\circ}$ lugar de venda entre as flores.

$O$ presente trabalho tem por finalidade aquilatar o crescimento e a absorção de $\mathrm{N}, \mathrm{P}, \mathrm{K}, \mathrm{Ca}, \mathrm{Mg}, \mathrm{S}, \mathrm{B}, \mathrm{Cu}, \mathrm{Fe}, \mathrm{Mn}$ e $\mathrm{Zn}$ pela rainha margarida cultivada em condições comerciais.

\section{MATERIAL E MÉTODOS}

Plântulas de rainha-margarida (Callestephus chinesis) foram transplantadas para canteiros no espaçamento de $0,2 \times 0,3 \mathrm{~m}$, na razão de 16 plantas por metro quadrado. Os canteiros receberam anteriormente 20 litros de esterco de curral, $170 \mathrm{~g}$ de superfosfato simples $\left(19 \% \mathrm{P}_{2} \mathrm{O}_{5}\right)$ e $30 \mathrm{~g}$ de cloreto de potássio $\left(62 \% \mathrm{~K}_{2} \mathrm{O}\right)$ por metro quadrado. Posteriormente, foram aplicadas $80 \mathrm{~g}$ de fosfato diamônico por metro quadrado, em quatro épocas diversas.

As épocas de amostragens do material vegetal, em número de seis, foram feitas inicialmente quando por ocasião do transplante e 18, 34, 46, 59 e 77 dias após. 0 número de plantas variou, de forma a se obter material suficiente para as análises químicas, mas nunca inferior a quatro plantas por amostragem. As plantas colhidas, foram lavadas para a determinação dos micronutrientes, divididas em raiz, caule, folhas e flores. As partes divididas foram secas em estufa a $85^{\circ} \mathrm{C}$ e analisadas para $\mathrm{N}, \mathrm{P}, \mathrm{K}, \mathrm{Ca}, \mathrm{Mg}$, $\mathrm{S}, \mathrm{B}, \mathrm{Cu}, \mathrm{Mn}$ eZn por métodos rotineiros adotados na análise de material vegetal (SARRUGE \& HAAG, 1974).

\section{RESULTADOS E DISCUSSAO}

\section{Crescimento}

O crescimento da rainha margarida expresso em aumento de peso na matéria seca, acha-se exposto no Quadro 1. Observa-se que o crescimento é lento até aos 34 dias após o transplante, cerca de $4,61 \%$ do total aos 77 dias. Dos 46 dias em diante ocorre um aumento acentuado, praticamente linear, no crescimento, com o aparecimento dos botões florais e com consequente desenvolvimento em flores aos 77 dias. Interessante observar que em 18 dias (59 dias após o transplante) o peso de matéria seca praticamente dobra. 


\section{Concentração e variação na quantidade dos macronutrientes}

Os teores percentuais e quantidades dos macronutrientes encontrados nas diversas partes da planta nos diversos estádios de desenvolvimento, acham-se assinalados no Quadro 2. Observa-se que, a concentração de $\mathrm{N}$, aumenta na raiz e no caule até aos 34 dias para posteriormente decrescer. Nas folhas, nos botões florais e nas flores este elemento praticamente não sofre oscilações com o desenvolvimento da planta. Chama atenção a concentração elevada de $\mathrm{N}$ nas folhas. A concentração de $\mathrm{P}$ aumenta nas raízes até aos 34 dias, para decrescer após este período; no caule a concentração decresce com a idade da planta. Nas folhas, botões florais e flores pouca oscilação apresenta o fósforo. O mesmo fenômeno é observado para o potássio $(\mathrm{K})$. A concentração de Ca decresce lentamente nas diversas partes da planta em função do crescimento, com exceção dos botões florais onde se estabiliza. $\mathrm{O}$ teor de $\mathrm{Mg}$ decresce nas raízes e no caule; sendo que permanece aproximadamente constante nas folhas e nas flores. A concentração de $\mathrm{S}$ decresce nas raízes, no caule e nas folhas. Nos botões florais e nas flores a concentração deste macronutriente mantem-se contante.

Considerando-se, o período, que antecede ao aparecimento dos botões florais, como sendo momento em que a planta tem maior necessidade de ter a sua disposição os nutrientes, os valores encontrados nas folhas dão uma indicação ao estado nutricional da mesma. Assim, os valores encontrados nas folhas, expressos em função da matéria seca foram: N\% 4,09-4,40; $\mathrm{P} \%$ 0,44-0,46; K\% 1,65-3,19; Ca\% 1,01-1,10; $\mathrm{Mg} \%$ 0,34-0,45; $\mathrm{S} \%$ 0,42-0,43.

0 Quadro 2 apresenta também, as quantidades dos macronutrientes extraídos, pela rainha margarida nos diferentes estágios de seu desenvolvimento. Observa-se que nos primeiros 18 dias após o transplante a extração dos nutrientes é baixa, como é de se esperar, cerca de $5 \%$ do total como pode ser visto no Quadro 3. A extração dos nutrientes acentua-se a partir desta idade para atingir em torno de $50 \%$ aos 59 dias após o transplante, com exceção do $\mathrm{P}$ que alcança 80,6\% do total (Quadro 3). Estes dados sugerem a vantagem de uma aplicação de fertilizantes, em cobertura no período compreendido entre 20 e 40 dias após o transplante.

Nos 18 dias restantes ao aparecimento das flores, ocorre uma intensa absorção de todos os macronutrientes, principalmente do $\mathrm{N}$ seguido pelo $\mathrm{Mg}, \mathrm{K}, \mathrm{S}$, Ca e finalmente do $\mathrm{P}$.

A rainha margarida pode ser considerada uma planta exigente em nutrientes quando em confronto com o cravo e gladíolo, como mostram os dados a seguir: 


\begin{tabular}{lcrrrrll}
\hline & \multicolumn{7}{c}{$\mathrm{mg} / \mathrm{planta}$} \\
\cline { 2 - 6 } Espécie & $\mathrm{N}$ & $\mathrm{P}$ & $\mathrm{K}$ & $\mathrm{Ca}$ & $\mathrm{Mg}$ & $\mathrm{S}$ literatura \\
\hline $\begin{array}{l}\text { gladíolo } \\
\text { cravo }\end{array}$ & 362 & 78 & 586 & 78 & 27 & 35 HAAG et al. (1970) \\
$\begin{array}{l}\text { rainha- } \\
\text {-margarida }\end{array}$ & 2.043 & 81 & 1.026 & 348 & 90 & 55 FERNANDES et al. (1971) \\
\hline
\end{tabular}

Considerando-se uma população de 110.000 plantas por hectare a cultura da rainha margarida extrae em ordem decrescente:

$$
\begin{array}{r}
\mathrm{K}-274,6 \mathrm{~kg} \\
\mathrm{~N}-225,5 \mathrm{~kg} \\
\mathrm{Ca}-100,7 \mathrm{~kg} \\
\mathrm{Mg}-39,2 \mathrm{~kg} \\
\mathrm{P}-23,3 \mathrm{~kg} \\
\mathrm{~S}-17,5 \mathrm{~kg}
\end{array}
$$

\section{Concentração e variação na quantidade dos micronutrientes}

As concentrações em ppm e as quantidades dos micronutrientes encontrados nas diversas partes da planta em função do crescimento acham-se expostas no Quadro 4. Observa-se que a concentração de B decresce na raiz, caule e folhas com a idade da planta. Nos botões florais e nas flores o teor deste micronutriente aumenta, domonstrando que houve translocação. A concentração de $\mathrm{Cu}$ decresce nas raízes, caule e folhas, mantendo-se aproximadamente constante nas folhas e nas flores. A concentração de Fe oscila na raiz e decresce lentamente nas demais partes da planta com o progredir do tempo. A concentração de $\mathrm{Mn}$ e $\mathrm{Zn}$ decresce em todas as partes da planta a medida que a mesma envelhece.

Do mesmo modo que para os macronutrientes a rainha margarida apresenta maior concentração dos micronutrientes estudados, na fase de anteceder o florescimento, isto é, dos 20 aos 40 dias após o transplnate.

Os valores encontrados nas folhas nesta fase foram: B ppm 23-36; Cu ppm 18-20; Fe ppm 105-150; Mn ppm 115-132; Zn ppm 64-111.

0 quadro 4 assinala igualmente as quantidades dos micronutrientes extraídos pela planta nos diversos estádios de desenvolvimento. Observa-se, que a quantidade absorvida até aos 34 dias após o transplante é pequena. Desta fase em diante acentua-se grandemente a absorção, atingindo porcentagem acima de $60 \%$ para o $\mathrm{B}, \mathrm{Mn}, \mathrm{Zn}$ e valores inferiores a $50 \%$ para $\mathrm{Cu}$ e Fe (Quadro 3). Nos últimos 18 dias a planta absorve cerca de $70 \%$ do $\mathrm{Cu}$ e $\mathrm{Fe}$ que apresentará com 77 dias de idade. 
Uma plantação de um ha de rainha margarida com uma população de 110.000 plantas extrae aproximadamente as seguintes quantidades de micronutrientes, em ordem decrescente:

$$
\begin{gathered}
\mathrm{Fe}-1.885 \mathrm{~g} \\
\mathrm{Mn}-763 \mathrm{~g} \\
\mathrm{Zn}-432 \mathrm{~g} \\
\mathrm{Cu}-337 \mathrm{~g} \\
\mathrm{~B}-238 \mathrm{~g}
\end{gathered}
$$

A rainha margarida absorve maior quantidade de micro-nutrientes por planta em confronto com o gladiolo, assim:

\begin{tabular}{lccccc}
\hline Espécie & $\mathrm{B}$ & $\mathrm{Cu}$ & $\mathrm{Fe}$ & $\mathrm{Mn}$ & $\mathrm{Zn}$ literatura \\
\hline $\begin{array}{l}\text { gladiolo } \\
\text { rainha- } \\
\text {-margarida }\end{array}$ & 1.019 & 95 & 1.655 & 914 & 272 HAAG et al. (1970) \\
\hline
\end{tabular}

\section{CONCLUSÕES}

a) o crescimento em peso de matéria seca é contínuo até o florescimento, acentuando-se a partir dos 46 dias.

b) os macronutrientes são absorvidos na seguinte ordem decrescente por planta: $2.496,6 \mathrm{mg} \mathrm{K} ; 2.049,9 \mathrm{mg} \mathrm{N} ; 915,7 \mathrm{mg} \mathrm{Ca} ; 356,6$ $\mathrm{mg} \mathrm{Mg} ; 159,1 \mathrm{mg} \mathrm{S} ; 212,5 \mathrm{mg} \mathrm{P}$.

c) os micronutrientes são absorvidos na seguinte ordem decrescente por planta: 17.142 ug $\mathrm{Fe}$; 6.946 ug $\mathrm{Mn}$; 3.931 ug $\mathrm{Zn}$; 3.070 ug $\mathrm{Cu}$; 2.140 ug B.

d) a rainha margarida é uma planta exigente em nutrientes.

MINERAL NUTRITION OF ORNAMENTAL PLANTS. III. ABSORPTION OF NUTRIENTS BY ASTER (Callestephus chinensis) PLANTS.

\section{SUMMARY}

Aster plants were harvested periodically at $0,18,34,46,59$ and 77 days after transplanting. The plants were divided into root, stem, leaves, flowers and analyzed for $\mathrm{N}, \mathrm{P}, \mathrm{K}, \mathrm{Ca}, \mathrm{Mg}, \mathrm{S}, \mathrm{B}, \mathrm{Cu}, \mathrm{Fe}, \mathrm{Mn}, \mathrm{Zn}$. 
It was observed that aster plant show a fast growth period after 46 days. An aster plant presents at 77 days old $2,049 \mathrm{mg} \mathrm{N} ; 212 \mathrm{mg} \mathrm{P}$; 2,496 mg K; $915 \mathrm{mg} \mathrm{Ca} ; 356 \mathrm{mg} \mathrm{Mg;} 159 \mathrm{mg} \mathrm{S} ; 2,140$ ug B; 3,070 ug Cu; 17,142 ug Fe; 6.946 ug $\mathrm{Mn}$ and 3,931 ug $\mathrm{Zn}$.

\section{LITERATURA CITADA}

FERNANDES, P. D., S. WATANABE, G. D. DE OLIVEIRA, H. P. HAAG. 1971. Nutrição Mineral de Plantas Ornamentais. II Absorção de Macronutrientes pelo Craveiro. Anais E. S. A. «Luiz de Queiroz» XXVIII : 135-143.

FERNANDES, P. D. 1974. Alguns Estudos de Adubação Mineral do Gladiolo (Gladiolus communis, L., cv. «Perusi»). Tese de Doutoramento. E. S. A. \&Luiz de Queiroz», USP, Piracicaba.

HAAG, H. P., G. D. DE OLIVEIRA, J. R. MATTOS. 1970. Nutrição Mineral de Plantas Ornamentais. I. Absorção de Nutrientes pela Cultura de Gladiolos. Anais E. S. A. «Luiz de Queiroz» XXVII : 125-141.

MIRANDA, M. A. L. S/data. Floricultura. Diagnóstico da Situação. Medidas Corretivas. Coordenadoria de Assistência Técnica Integral «CATI». Campinas.

SARRUGE, J. R., H. P. HAAG. 1974. Análises Químicas em Plantas. Departamento de Química, E. S. A. «Luiz de Queircz», USP. Piracicaba. 
QUADRO 1 - Peso da matéria seca $(g)$ e porcentagem de crescimento em função do estádio de desenvolvimento da planta Média mínima de 4 plantas por amostragem.

\begin{tabular}{|c|c|c|c|}
\hline $\begin{array}{c}\text { Dias após } \\
\text { transplante }\end{array}$ & Parte da planta & $\begin{array}{l}\text { Peso de mat. } \\
\text { seca (g) }\end{array}$ & $\begin{array}{l}\text { Porcentagem de } \\
\text { crescimento }\end{array}$ \\
\hline & raiz & 0,23 & \\
\hline \multirow[t]{4}{*}{0} & caulo & 0,10 & \\
\hline & folhas & 1,18 & \\
\hline & total & 1,57 & 1,21 \\
\hline & raiz & 0,33 & \\
\hline \multirow[t]{4}{*}{18} & caule & 0,42 & \\
\hline & folhas & 1,70 & \\
\hline & total & 2,45 & 1,89 \\
\hline & raiz & 0,62 & \\
\hline \multirow[t]{4}{*}{34} & caule & 1,29 & \\
\hline & folhas & 4,06 & \\
\hline & total & 5,97 & 4,61 \\
\hline & raiz & 2,80 & \\
\hline \multirow[t]{5}{*}{46} & caule & 15,85 & \\
\hline & folhas & 19,89 & \\
\hline & botóes fl. & 0,82 & \\
\hline & total & 39,36 & 30,40 \\
\hline & raiz & 3,09 & \\
\hline \multirow[t]{5}{*}{59} & caule & 38,33 & \\
\hline & folhas & 15,21 & \\
\hline & botões fl. & 11,25 & \\
\hline & total & 67,88 & 52,44 \\
\hline & raiz & 6,27 & \\
\hline \multirow[t]{5}{*}{77} & caule & 64,70 & \\
\hline & folhas & 22,6 & \\
\hline & flores & 35,91 & \\
\hline & total & 129,44 & 100,00 \\
\hline & folhas & 1,70 & \\
\hline
\end{tabular}




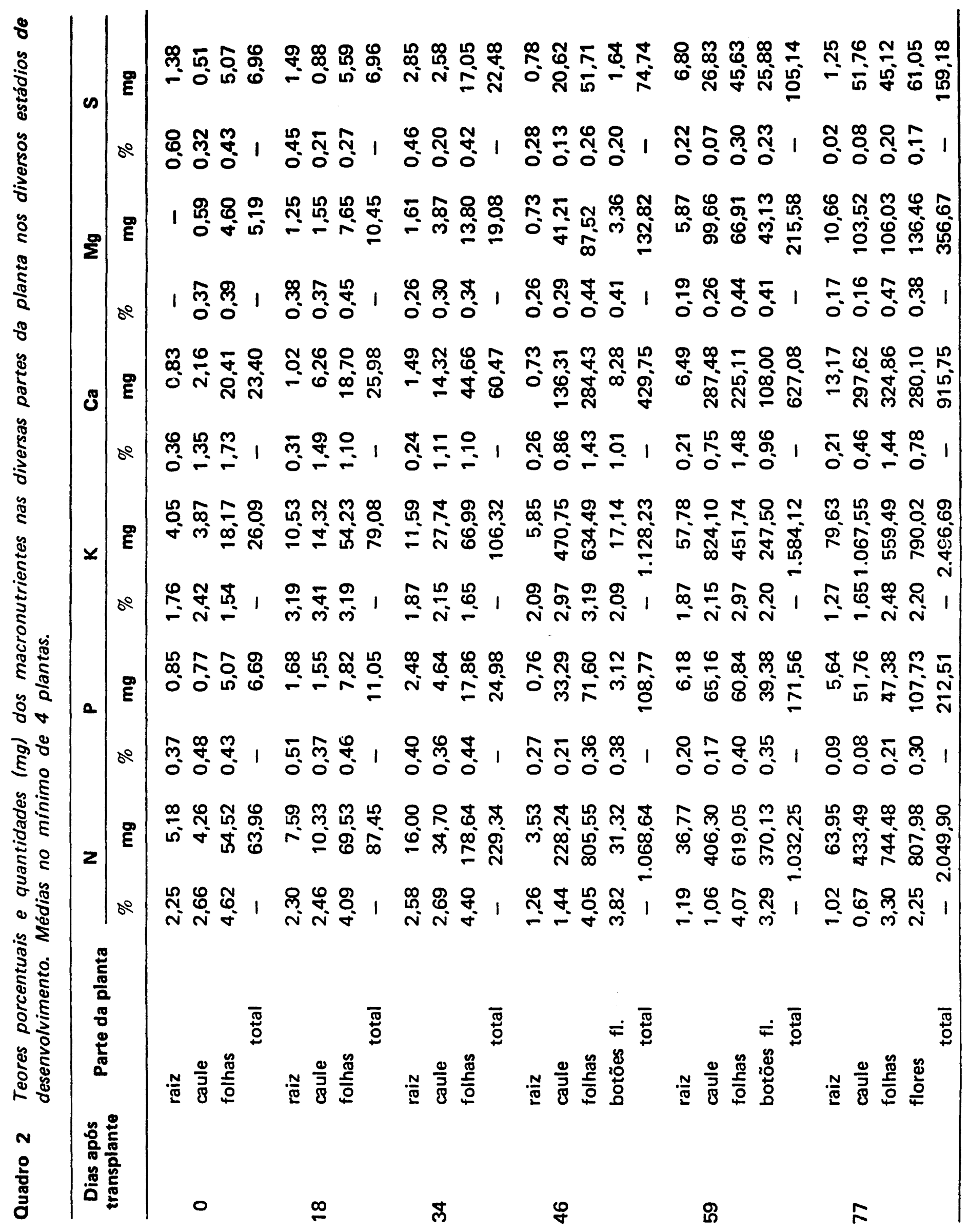




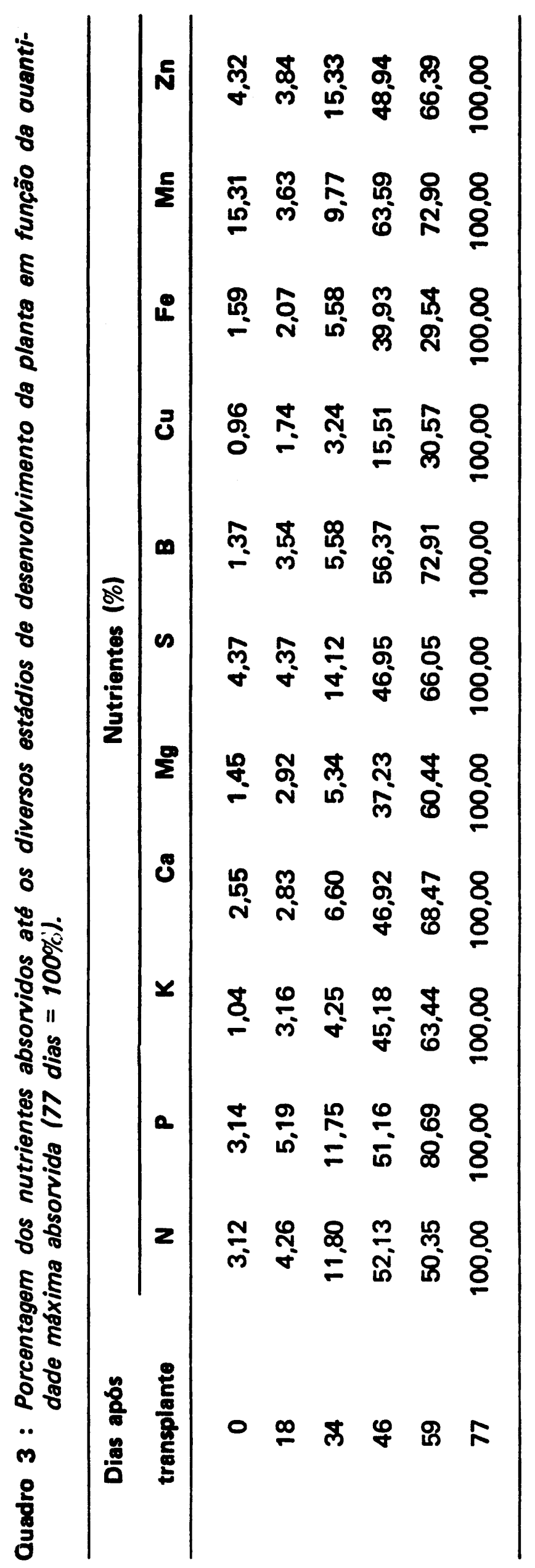




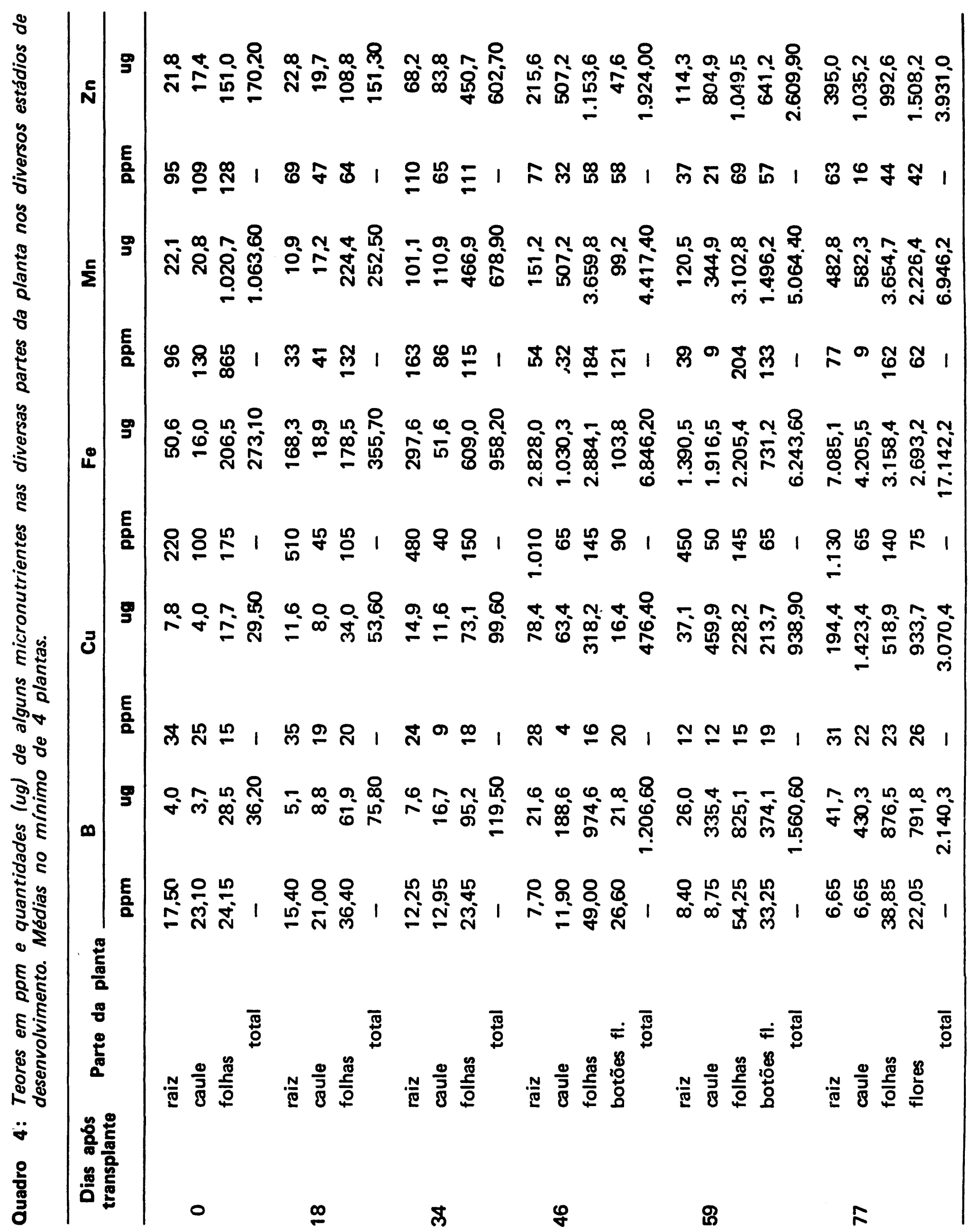

